\title{
Quality of Service (QoS) based Service Retrieval Engine
}

\author{
Hai Dong \\ Digital Ecosystems and Business \\ Intelligence Institute \\ Curtin University of Technology \\ Perth, Australia \\ +61892667085 \\ hai.dong@cbs.curtin.edu.au
}

\author{
Farookh Khadeer Hussain \\ Digital Ecosystems and Business \\ Intelligence Institute \\ Curtin University of Technology \\ Perth, Australia \\ +61892669298
}

farookh.hussain@cbs.curtin.edu.au

\author{
Elizabeth Chang \\ Digital Ecosystems and Business \\ Intelligence Institute \\ Curtin University of Technology \\ Perth, Australia \\ +61892661235
}

elizabeth.chang@cbs.curtin.edu.au

\begin{abstract}
It is observed that there are few service evaluation and ranking methodologies currently available in the SOE. In this paper, we propose an innovative service evaluation and ranking strategy, based on the measurement of trustworthiness and reputation of services (or service providers'). CCCI Metrics originally proposed and developed by Chang et al [1] is used to measure the trustworthiness and reputation of e-services. Here we extend the application of CCCI Metrics to the field of service retrieval. A java-based search engine prototype is designed, with the purpose of implementing the trustworthiness and reputation-based service search, evaluation and ranking. Conclusions and future works are drawn in the final section.
\end{abstract}

Categories and Subject Descriptors

D.2.8 [Software Engineering]: Metrics - product metrics. H.3.5 [Information Storage and Retrieval]: Online Information Services - web-based services.

\section{General Terms}

Algorithms, Measurement, Design, Experimentation, Standardization, Theory

\section{Keywords}

CCCI Metrics, quality of service, reputation evaluation, serviceoriented environment, service ranking, trustworthiness evaluation

\section{INTRODUCTION}

Service Oriented Environment (SOE) is defined as a collaborative, shared and open environment which provides agents with infrastructures and technologies to carry out business services [1]. In this paper, we propose a quality of service (QoS) based service retrieval and ranking methodology for the SOE.

Permission to make digital or hard copies of all or part of this work for personal or classroom use is granted without fee provided that copies are not made or distributed for profit or commercial advantage and that copies bear this notice and the full citation on the first page. To copy otherwise, or republish, to post on servers or to redistribute to lists, requires prior specific permission and/or a fee

iiWAS 2008, November 24-26, 2008, Linz, Austria.

(c) 2008 ACM 978-1-60558-269-6/08/0011 \$5.00.
The paper is organized as follows:

First of all, we will make a brief survey of the existing service evaluation and ranking systems and analyze the problems in the current research. This is explained and done in Section 2. Next, we employ a case study to illustrate the need rank the service search results as per the Quality of Service provided. (QoS). We make use of the CCCI metrics, which have been proposed by Chang et al [1], in order to rank the retrieved services according to the Quality of Service (QoS). The application and working of the CCCI metrics for service search retrieval process in explained in Section 3. To evaluate the methodology, we implement a prototype of a service search, evaluation and ranking system. This is explained in Section 4. Finally, in Section 5, we conclude the paper and provide directions for future work.

\section{SERVICE EVALUATION AND RANKING SYSTEMS}

While a great amount of literature focuses on evaluating quality of services (QoS), few of them study on the integration of service evaluation and service ranking system, which is extremely relevant in any service search retrieval process.

Toma et al [4] propose a web service ranking system based on two different ranking strategies. One strategy is to use the Web Service Modeling Ontology (WSMO) to describe the values of Non-Functional Properties (NFPs) of web services, such as QoS, Service Level Agreement (SLA) etc. Hence web services can be ranked according to the values of user-preferred NFP. Another strategy is a multi-criteria ranking, which considers ranking multiple NFPs from three main perspectives - the user-preferred NFPs, the level of importance of the NFPs, and the ascending or descending order of services [4].

Gekas et al [3] propose a set of metrics for web service ranking Four main categories of ranking strategies are provided by these metrics, which are degree-based rankings that calculate the percentage of fed services in each web service, hubs-authoritiesbased rankings that calculate the ratio between the number of incoming services and the number of outgoing services, nonfunctional rankings that focus on the NFPs of web service, and non-connectivity rankings that focus on the connectivity of web service networks [3]. 
The limitations of these service ranking systems can be concluded as follows:

- They are all designed for the web service environment, which cannot adapt to the broader and more complicated SOE.

- Despite the fact that they are all equipped with the QoS ranking methodology; none of them integrate their ranking system with their corresponding QoS evaluation systems.

- None of them consider the factor of trustworthiness and reputation in the service ranking.

Against the backdrop of the above limitations, in Section 3, we explain how the application of CCCI metrics could be used for service search ranking process.

\section{Application of CCCI metrics for service search retrieval}

CCCI Metrics is a group of metrics developed by Chang et al [1], with the purpose of measuring the Quality of Service (QoS) provided by a given service provider [1] [2]. These metrics are grounded on the assumption that a service interaction involves at least one criterion. A service can be regarded of as an ordered set of criteria. A criterion is defined as a decisive factor of the mutually agreed service performance between the service provider and service requester [1]. The service requester can evaluate the performance of the service provider in each of the decisive factors after the service interaction. Subsequently, the issue of determining the Quality of Service (QoS) comes down to the issue of measuring and quantifying the service delivered in each individual criterion and aggregating them.

We would make use of the proposed CCCI metrics and applying them in the scenario of service search retrieval in order to rank these service providers. The working of the proposed application of CCCI metrics in the domain of service search retrieval is explained in the rest of this section with the help of a case study. It is important to note that in the context of this paper it is not possible to explain the working of the $\mathrm{CCCI}$ metrics in detail. We would like to encourage interested reader to refer to Chang et al $[1,2]$ for an in-depth explanation of the working of CCCI metrics.

Hai lives in City $A$ and one day he needs a taxi service. There are many companies that provide taxi services within City A. As a result, Hai intends to find out the overall QoS ranking for the taxi companies in order to make an objective decision. In addition, Hai may intend to make a service selection by assigning more preference to certain criteria.

The metrics proposed within the framework of CCCI Metrics' which would help the service requestor (Hai) to rank the service providers are as follows:

- To find out the reputation of the companies that provide taxi services in City A from the perspective of taxi services reputation;
- To find out the performance of the companies in each industry standard (criterion) of taxi services $-A B$ Corr $_{\text {Criterion; }}$;

- To evaluate Hai's trustworthiness of a taxi service trustworthiness;

- to evaluate its performance in each taxi industry standard (criterion) $-A B$ Corr $_{\text {Criterion }}$

- To evaluate the clarity of each taxi service industry standard (criterion) - Clear $_{\text {Criterion }}$

- To evaluate the importance of each taxi service industry standard (criterion) - Imp $p_{\text {Criterion }}$

We have designed a semantic service search engine which can reason semantically and retrieve services providers which can deliver on the tasks described in the search query. The steps that the service requestor employs are as follows:

- A service requester uses the search system GUI to query for a given service providers or a group of service providers that can deliver on the tasks described in the search query. The search process is semantic (shown in Figure 1)

- The retrieved service providers can be either ranked according to their reputation values or actual behavior values for a given criterion (ActualBehaviour Criterion )

- After a service provider is chosen and the service interaction with the service provider is completed, the service requester, in its role as the trusting agent evaluates the provided Quality of Service (QoS). Our system provides then leads the service requestor to a GUI via which it can assign values to each of the following metrics for each criterion involved in the service interaction: $A B C o r r_{\text {Criterion, }}$ Clear $r_{\text {Criterion, }}$ and Imp Criterion

- After the evaluation is submitted to the system, the trustworthiness value of the service requester and the reputation of the service provider are recomputed and updated. Additionally the ActualBehaviour Criterion value of each criterion with regard to the service is recomputed. These two updated parameters are used to rank carry on the service search retrieval process.

\section{Implementation of CCCI Metrics for service search retrieval process}

In this section, we will explain the implementation of the $\mathrm{CCCl}$ Metrics in the service search ranking.

To evaluate the conceptual model of the extended CCCI Metricsbased service search, ranking and evaluation system, we implement a prototype by using Java and MySQL. The data source of the services stored in this system is obtained from the Australian Yellowpages ${ }^{\circledR}$ website (www.yellowpages.com.au). Figures 1 and 2 are the screenshots of the prototype, which is described in the rest of this section. 
Figure 1 reveals the service search and ranking function of the system. Once a service requester (e.g. User id: Hai) logs in the system and enters a service search query (e.g. taxi) in the search engine GUI, the retrieved service providers with respect to the service are retrieved and indexed by their reputation values or

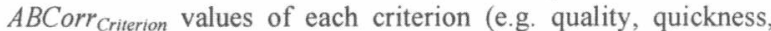
and price) involved in the service. From figure 1, we can see that as the user has not completed a service interaction with the selected service provider (Airport Executive Chauffer), (s)he is not eligible to evaluate the service provider's reputation with respect to the service.

Figure 2 reveals the service evaluation function of the system After the service requester completes a service interaction with the selected service provider, the service requester obtains the permission to evaluate the service provider, which enables the service requester to access a service evaluation form with respect to the service provider. After assigning values to the metric of $A B$ Corr $_{\text {Criterion (labeled as "Your evaluation"), Clear }}$ Criterion (labeled as "Clarity"), and Imp $p_{\text {Criterion (labeled as "Importance") }}$ for each criterion in this form, the service provider can click the "Submit" button. Once the form is submitted, the trustworthiness value that the service requestor has in the service provider will be shown in the "Trustworthiness" area in the form. Then the $A B C o r r_{\text {Criterion }}$ value of each criterion and the reputation value of the service provider will be updated, which can be used for further ranking purposes. It needs to be noted that a service requester only has one opportunity of evaluation after a service interaction. In other words, once the service requester submits the evaluation form, s/he cannot change or resubmit it any more.

\section{CONCLUSIONS AND FUTURE WORKS}

In this paper, we introduce a trustworthiness and reputation-based service evaluation and ranking methodology in the SOE, against the drawback that there is few available and integrated evaluation and ranking methodologies in this field. This methodology is based on the CCCI Metrics developed by Chang and Hussain CCCI metrics is built upon the assumption that each service interaction has at least one criterion. Hence, the measurement of a service interaction turns into the measurement of each criterion involved in the service interaction. $\mathrm{CCCl}$ Metrics consists of four primary parameters - correlation of an interaction ( Corr $\left._{\text {Interaction }}\right)$, correlation of a criterion $\left(\right.$ Corr $\left._{\text {Criterion }}\right)$, clarity of a criterion $\left(\right.$ Clear $\left._{\text {Criterion }}\right)$ and importance of a criterion $\left(\operatorname{Imp}_{\text {Criterion }}\right)$. The trustworthiness value of a service interaction would be same as

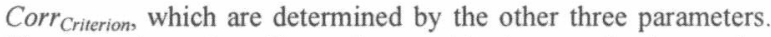
The reputation value of a service provider in a service interaction is determined as the average of all involved third party recommendation agents' trustworthiness values towards this service provider in the service interaction. Thus, a service provider's reputation value in a service interaction can be used as a ranking factor. In addition, we provide the concept of actual behavior of a service provider in a criterion (ActualBehaviour $_{\text {Criterion) }}$ ) for service requesters to rank service providers according to a given criterion. To validate the methodology, we implement the prototype of the service search, evaluation and ranking system, which reveals the whole workflow of the proposed system.

In the future, we will work on the multiple ranking strategies other than trustworthiness and reputation, to enhance the function of service evaluation and ranking in SOE. Currently we are carrying out the study on integrating the factor of user preference into our ranking prototype, by using the technology of ontologies.

\section{ACKNOWLEDGMENTS}

We would like to express our gratitude to the assistance of all relevant DEBII staff, especially to our programmer Wei Liu who took the responsibility of implementing the prototype of the service search, evaluation and ranking system.

\section{REFERENCES}

[1] E. Chang, T. S. Dillon, and F. Hussain, Trust and Reputation for Service Oriented Environments-Technologies for Building Business Intelligence and Consumer Confidence: John Wiley \& Sons, 2005.

[2] E. Chang, F. K. Hussain, and T. S. Dillon, "CCCI Metrics for the measurement of quality of e-service," in the 2005 IEEE/WIC/ACM International Conference on Intelligent Agent Technology (IAT'05), France, 2005. DOI= http:/dx doi org/10.1109/LAT 2005.51

[3] J. Gekas, "Web service ranking in service networks," in the 3rd European Semantic Web Conference (ESWC 2006), Budva, 2006.2 DOI= http:/doi.ieecomputersociety. org/10.1109/1CWS.2004.1314 $\underline{739}$

[4] I. Toma, D. Roman, D. Fensel, B. Sapkota, and J. M. Gomez, "A multi-criteria service ranking approach based on nonfunctional properties rules evaluation," in ICSOC 2007, B. Kr"amer, K.-J. Lin, and P. Narasimhan, Eds. Heidelberg: Springer-Verlag, 2007, pp. 435-441. DOI $=10.1007 / 978-3-$ 540-74974-5 


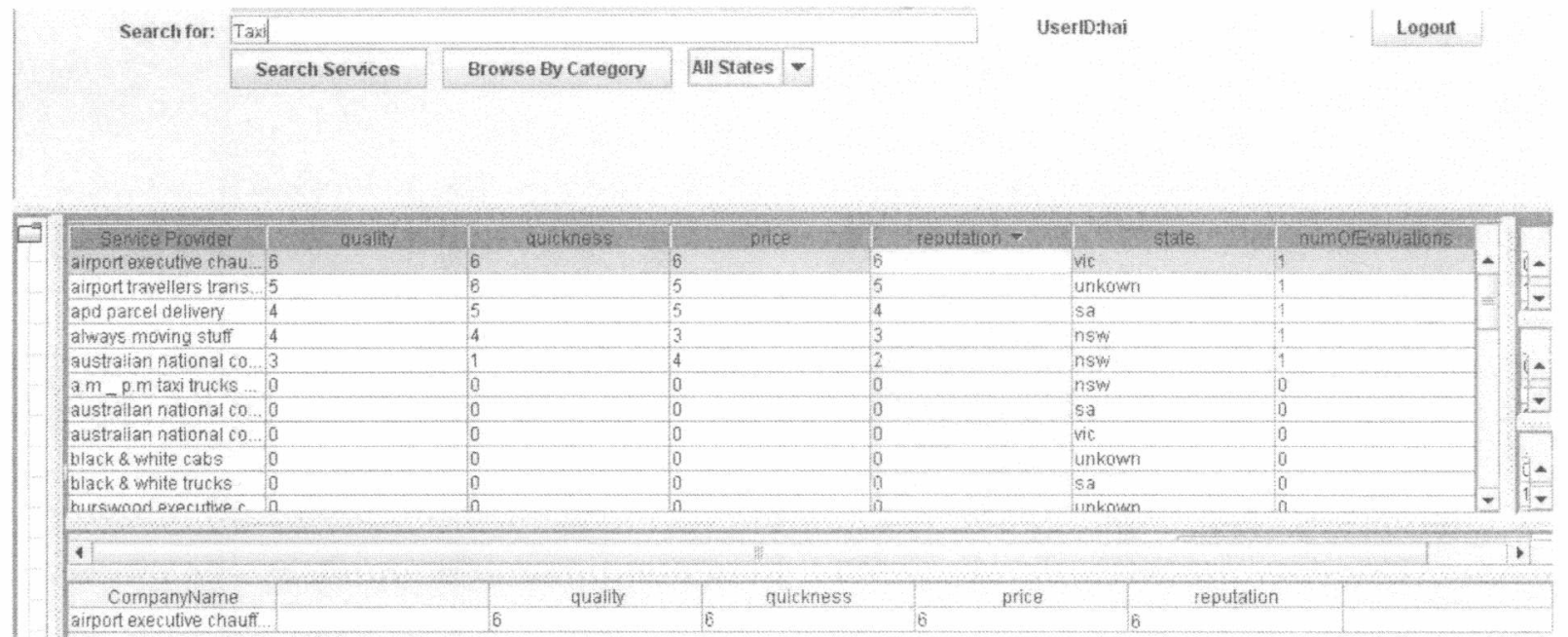

Figure 2. Screenshot of the service search and ranking

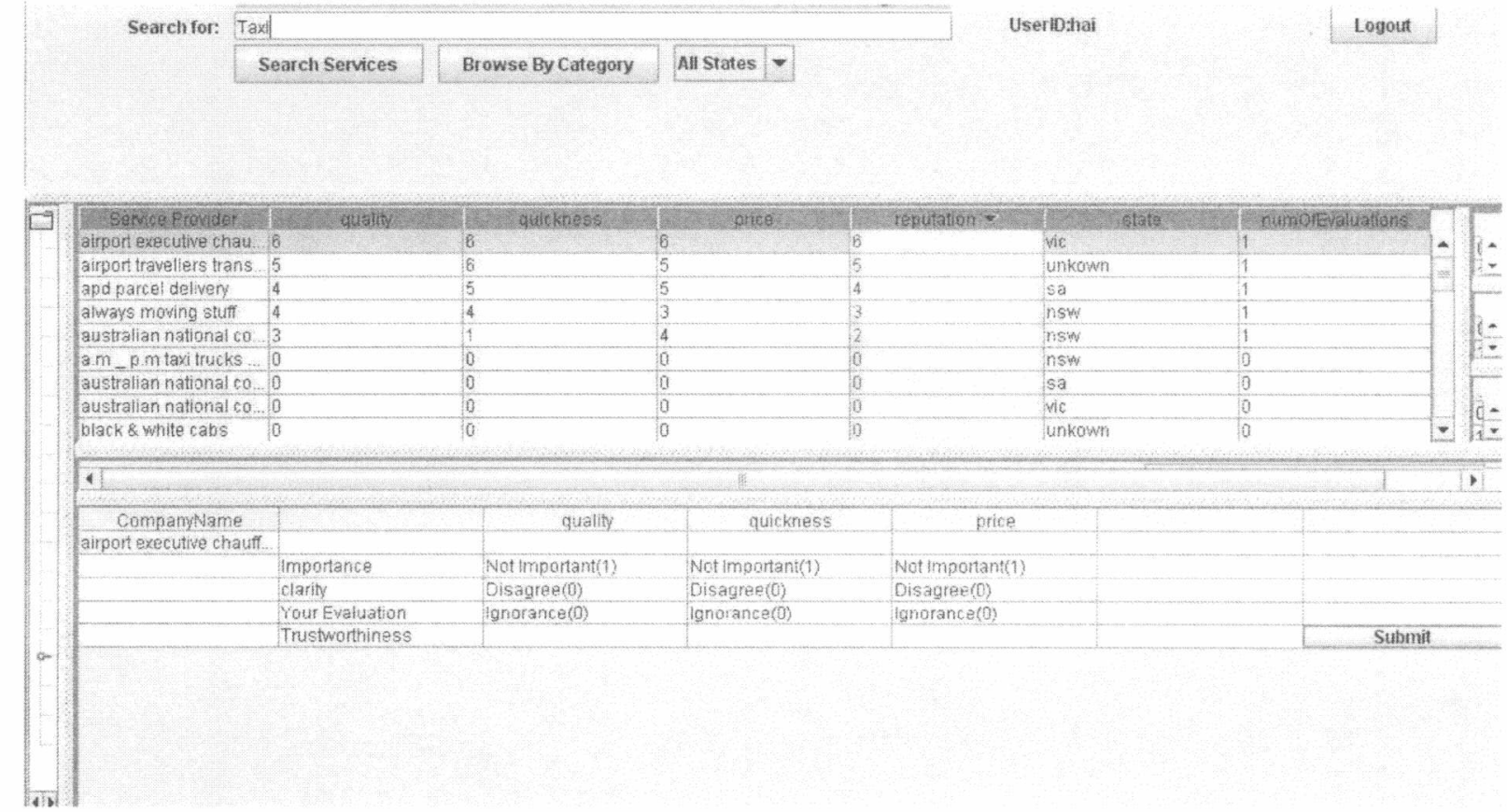

Figure 3. Screenshot of the service evaluation 